\title{
DIE DAA E. V. GEHT NEUE WEGE IN DER AUSBILDUNG!
}

\section{Liebe Kolleginnen, liebe Kollegen!}

WEBINARE | Aufgrund der bestehenden Einschränkung derVersammlungsfreiheit durch die Regelungen der Bundes- und Landesregierungen fallen immer wieder Präsenzveranstaltungen aus. Einen Ausweg aus dem Weiterbildungsstopp stellen Webinare dar. Trotz anfänglicher Bedenken, diesen Weg zu beschreiten, erschien uns doch dieVerpflichtung zur Fortführung der Akupunkturausbildung wichtiger als der Nachteil, der durch fehlende Präsenz in den Seminarkursen entsteht. Die Premiere fand am 25./26.April 2020 mit unserem Webinar „Einführung in die wissenschaftliche Akupunktur" statt. Da Audio- und Kameratechnik sowie digitaleVortragstechniken schon seit vielen Jahren in unseren Kursen in hoher Qualität zur Anwendung kommen, haben wir es mithilfe eines kreativen Technikers geschafft, unsere teilnehmenden Kollegen vollends zu begeistern. Mittels Audiofreigabe, Chat und Umfragen ist eine interaktive Unterrichtsgestaltung möglich. Der überwältigende Zuspruch hat uns ermutigt, weitere Webinare anzubieten. Informieren Sie sich dazu bitte auf unserer Homepage (www.akupunktur.de/seminare.html).

HOMEPAGE-START 27. APRIL 2020 | Die neue Homepage ist seit dem 27. April 2020 online.

„Gelungen!"

„Darauf habe ich lange gewartet!“

„Herzlichen Glückwunsch zu dieser schönen Seite!“

„, Gute Funktionalität - es macht Spaß sich auf

dieser Seite zu bewegen!"

„,Wirklich erstaunlich, was da entstanden ist! “

Das sind nur einige der vielen begeisterten Zuschriften, die uns in den letzten Wochen erreicht haben. Gerne nehmen wir dieses Lob nach den zwei intensiven Jahren der Entwicklung und Umsetzung entgegen. Dabei möchte ich an dieser Stelle den vielen kreativen und eifrigen Mitwir- kenden „Danke“ sagen. Die Firma Engels-MV-Management (Waren) hat mit schier unendlicher Geduld und viel Kreativität die Entwicklung der neuen Seite vorangebracht und das Design der Seite erstellt. Die Firma primaweb (Waren) hat die vielen Ideen mit ihren Programmierkünsten zum Leben erweckt. Danke möchte ich auch Herrn Ott von der Firma Mursoft (Graz) sagen. Durch seinen Einsatz wurde es möglich, die Inhalte im Mitgliederbereich für Sie individualisiert darzustellen. Zuletzt möchte ich aber auch dem Team in unserem Büro in München und da ganz besonders Frau Weber meinen Dank aussprechen. In unendlich vielen Stunden hat sie die Inhalte überarbeitet und eingestellt, die Korrekturen eingepflegt und in Zusammenarbeit mit den übrigen Beteiligten immer wieder nach notwendigen Lösungen gesucht.

SAVE THE DATE - GARMISCH 2021 | Leider mussten wir aus gegebenem Anlass die diesjährige Refresher- und TCM-Woche in Garmisch absagen, da das Hotel erst Anfang Juni öffnen durfte. Derzeit arbeiten wir an einer Lösung für den Refresher-Kurs (u.U.Webinar im August), um den vielen Kollegen mit dem Wunsch, das A-Diplom ablegen zu können, einen Vorbereitungskurs anbieten zu können. Die A-Diplomprüfung wird im Herbst in Timmendorf möglich sein. Die TCM-Prüfung wird auf das Jahr 2021 verschoben. Daher sollten Sie sich schon jetzt die Refresher- und TCM-Woche in Garmisch vom 07. bis 12. Mai 2021 vormerken.

Mit guten Wünschen für den Sommer,

Ihr

B. Ramme

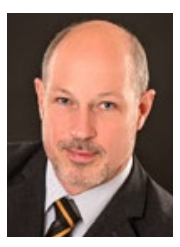

Dr. med. Bernd Ramme

1. Vorsitzender der DAA e.V.

Osserstraße 40, D-81679 München

Tel. $+4989 / 8145252$

E-Mail des allg.Büros: kontakt@akupunktur.de, Internet:www.akupunktur.de 